\title{
Greetings and many thanks from the desk of your Co-Editor-in-Chief
}

\author{
Claudio N. Soares
}

Published online: 9 March 2010

(C) Springer-Verlag 2010

As I have the opportunity to address our readers as your Co-Editor-in-Chief, I would like to first express our immense gratitude to all researchers, clinicians and scientists who have generously agreed to donate part of their precious time and acted as reviewers during 2009-a complete acknowledgement list can be found in this issue. As you all know, the AWMH has experienced an exponential increase in number of submissions; with such increase, a careful screening and reviewing process is absolutely necessary and we could not have achieved our goals to select high-quality, clinically meaningful manuscripts without the support of our referees - thank you all!

These are very exciting times for those who have long battled for a journal solely dedicated to Women's Mental Health. First, the impact factor of our journal has increased significantly over the past few years; second, we are promoting and consolidating "critical thinking" on issues related to women's mental health across the lifespan-for a sample of that, please refer to thoughtful considerations on the DSM-V approach to female-specific issues in mental health featured in the first issue of 2010. More importantly, the expertise of our Editorial Board and reviewers allows us to continue expanding the scope of our journal. As reflected in this issue of the AWMH, we are committed to publish clinical, epidemiologic and translational research that are innovative and may ultimately promote significant advances in the field of women's mental health-from menarche to menopause and beyond.

I hope you will enjoy this issue of the AWMH and many more to come. Please do not hesitate to share your critiques, thoughts, suggestions...our 'doors' are always open!

Yours truly,

Claudio N. Soares

Co-Editor-in-Chief

\section{N. Soares}

Department of Psychiatry and Behavioural Neurosciences, Mood Disorders Division, McMaster University,

Hamilton, ON, Canada

\section{N. Soares}

Department of Obstetrics and Gynecology,

McMaster University,

Hamilton, ON, Canada

C. N. Soares $(\bowtie)$

Women's Health Concerns Clinic, St. Joseph's Healthcare,

Hamilton, ON, Canada

e-mail: csoares@mcmaster.ca 